\title{
Prevalencia de Dependencia de Nicotina en Algunas Poblaciones: una Revisión Sistemática
}

\author{
Adalberto Campo-Arias \\ Médico, Especialista en Psiquiatría. Facultad de Medicina, Universidad Autónoma de Bucaramanga. E- \\ mail: acampoar@unab.edu.co
}

Recibido 30 Agosto 2005/Enviado para Modificación 5 Enero 2006/Aceptado 14 Febrero 2006

\section{RESUMEN}

En el mundo, el consumo de tabaco es la principal causa de morbi mortalidad prevenible. En la mayoría de los fumadores, la dependencia de nicotina (DN) explica que se siga en el consumo a pesar de los efectos nocivos sobre la salud. El objetivo de esta revisión fue establecer la prevalencia actual y anual de DN en personas fumadoras de algunas poblaciones. Se realizó una revisión sistemática en las bases de datos Ebsco, Embase, Lilacs, Ovid, Proquest y PubMed. Se incluyeron como palabras de búsqueda smoking, tobacco, cigarette, nicotine, dependence, adults, general population y crosssectional study. Se revisaron sólo estudios en español, inglés y portugués. Se realizó un análisis descriptivo de seis artículos que reunieron los criterios de inclusión. La prevalencia actual de DN estuvo entre 27,8 y $55 \%$ (promedio ponderado $44,7 \%$ ) y la prevalencia anual entre 26 y 46,9\% (promedio ponderado $37,7 \%$ ). La DN fue independiente del sexo y mayor en quienes fumaban un mayor número diario de cigarrillos. Se concluye que la DN se presenta en aproximada mente $45 \%$ de los fumadores regulares actuales y en el $38 \%$ de los fumadores durante el último año. Se necesita más investigación.

Palabras Clave: Tabaquismo, trastornos por uso de tabaco, adulto, revisión (fuente: DeCS, BIREME).

\section{ABSTRACT}

The prevalence of nicotine-dependency in some populations: a systematic review

Tobacco use is the leading cause of preventable morbidity-mortality around the world. Nicotine dependence (ND) explains why people continue to 
smoke even though the harmful outcome associated with tobacco use is well-known. The object of this review was to establish current and one-year prevalence of ND among adults from the general population. Ebsco, Embase, Lilacs, Ovid, Proquest, and PubMed were systematically reviewed. The key words used were: smoking, tobacco, cigarette, nicotine, dependence, adults, general population and cross-sectional study. Only research carried out in English, Portuguese and Spanish was reviewed. A descriptive analysis of six articles was made. Current ND prevalence ranged from 27,8 $\%$ to $55 \%(44,7 \%$ weighted average) and annual prevalence from $26 \%$ to $46,9 \%(37,7 \%$ weighted average). ND was independent of gender and higher amongst heavy smokers. It was concluded that ND prevalence is close to $45 \%$ amongst current regular smokers and affected $38 \%$ of smokers during the last year. More research is needed.

Key Words: Smoking, tobacco use disorder, prevalence, adult, review (source: MeSH, NLM).

E n el mundo, los comportamientos modificables se asocian con las principales causas de mortalidad (1). En este grupo de conductas, el consumo de cigarrillo explica el mayor número de muertes que pueden ser objeto de una activa prevención $(2,3)$. El consumo habitual de cigarrillo es responsable de un número significativo de años de vida potenciales perdidos y de otros costos sociales y económicos (4).

El consumo de cigarrillo es la forma más frecuente de uso de tabaco (5). En consecuencia, a lo largo de esta revisión se usará en forma indistinta consumo de cigarrillo y uso de tabaco. En el contexto mundial, varios estudios informan la prevalencia de consumo de cigarrillo en la población general, la prevalencia varía de una región a otra (6). En países del Medio Oriente como Arabia Sauditas se informan prevalencias tan bajas como 11 \% (7). En países de europeos como Estonia se encuentran prevalencias superiores al $40 \%$ (8). En Colombia, la prevalencia de consumo de cigarrillo durante el último mes alcanza el $21 \%$, en promedio, con variaciones regionales importantes (9). Por otro lado, la prevalencia actual de dependencia de nicotina (DN) se encuentra en el 0,1 \% y prevalencia anual en el 0,5\% de toda la población, cifras extraordinariamente bajas (10). Alrededor del mundo, existen menos datos disponibles en relación con la DN en las personas fumadoras. Se desconoce la prevalencia de DN en fumadores de la población general colombiana.

Es importante diferenciar consumo de cigarrillo (regular u ocasional) de la DN (11). No todos los fumadores regulares reúnen criterios para DN, según los criterios de la Clasificación Internacional de Enfermedades (CIE-10) 
de la Organización Mundial de la Salud (OMS) (12) y la cuarta versión revisada del Manual Diagnóstico y Estadístico (DSM-IV-TR) de la Asociación Psiquiátrica Americana (APA) (13). Las personas con dependencia presentan síntomas de abstinencia con la reducción en el número de cigarrillo fumados durante el día o con la suspensión total y tienen mucha dificultad para abandonar el consumo de cigarrillo (14). El primer criterio para DN es el consumo de un cigarrillo, al menos, todos los días durante el último mes $(12,13)$. Se acepta que el número diario de cigarrillos fumados es el mejor predictor DN (15). Es infrecuente el diagnóstico de DN en fumadores de menos de cinco cigarrillos por día (16). Las personas con un patrón de consumo regular de cigarrillo representan la población en mayor riesgo para las enfermedades relacionadas con el uso crónico de tabaco (17).

La identificación de fumadores habituales que presentan DN se puede realizar mediante la aplicación de cuestionarios autodiligenciados breves, como la escala de Fagerström para DN (18) o la escala de Etter para dependencia al cigarrillo (19). Sin embargo, el diagnóstico es más válido y confiable cuando se aplica una entrevista estructurada (20).

Es importante conocer la prevalencia actual y anual de DN en fumadores regulares de la población general. Los profesionales de la salud deben indagar la condición de fumador de todos los pacientes, ambulatorios y hospitalizados, e identificar los posibles casos de DN (21). La DN es otro aspecto a considerar en el momento de la promoción del abandono del consumo de cigarrillo. Los fumadores con DN necesitan, por lo general, una intervención farmacológica para mantener la abstinencia (22-24).

El objetivo de esta revisión es presentar la prevalencia actual y anual de DN, según los criterios de la APA en fumadores de algunas poblaciones, sin diagnóstico formal de enfermedades médicas o trastornos mentales, mediante el uso de entrevista estructurada.

\section{MÉTODOS}

Para esta revisión sistemática se realizó una búsqueda en las bases de datos de Ebsco, Embase, Lilacs, Ovid, ProQuest y PubMed. Se incluyeron como palabras de búsqueda en todas las combinaciones posibles los términos MeSH (Medical Subject Heading): smoking, tobacco, cigarette, nicotine, dependence, prevalence y cross-sectional study. Este proceso se complementó con una revisión directa de cada una de las referencias que citaban de las publicaciones encontradas en las bases de datos para identificar artículos que pudieran haberse omitidos en la búsqueda inicial. 
Se seleccionaron los artículos publicados originales desde enero de 1990 hasta julio de 2005 en los cuales se informaba la prevalencia actual (consumo de cigarrillo durante el último mes) o anual (consumo de cigarrillo durante el último año) de DN en fumadores de algunas poblaciones, sin diagnóstico formal de enfermedades médicas o trastornos mentales. Se incluyeron sólo los artículos en los cuales el diagnóstico de DN se realizó con una entrevista clínica estructurada aplicada por un profesional de la salud mental o un encuestador entrenado sin formación médica. Se excluyeron aquellos estudios que informaban la DN a partir de la aplicación de un instrumento de tamización como la escala de Fagerström para nicotina o la escala de Etter para dependencia al cigarrillo. Las escalas son instrumentos para identificar posibles casos y no confirmaron un diagnóstico clínico como la DN (20).

Se realizó un análisis descriptivo, se anotaron las características sociodemográficas de las poblaciones y la prevalencia actual y anual de DN. Se calculó la prevalencia ponderada para tener un promedio estimado global. A la prevalencia actual y anual ponderada se le halló un intervalo de confianza del 95 \%. No se hizo clasificación del nivel de evidencia.

\section{RESULTADOS}

Ocho artículos resultados de investigaciones transversales reunieron los criterios de inclusión. Estos informes correspondían a seis estudios (25-32). Tres informes correspondían al mismo estudio (25-27). Finalmente, se analizaron tres estudios que informaban la prevalencia actual (25-29) y tres la prevalencia anual (30-32) de DN. Las características de estos estudios y las prevalencias se presentan en la Tabla 1.

Tabla 1. Características de los estudios que informan la prevalencia actual y anual de dependencia de nicotina en personas de algunas poblaciones

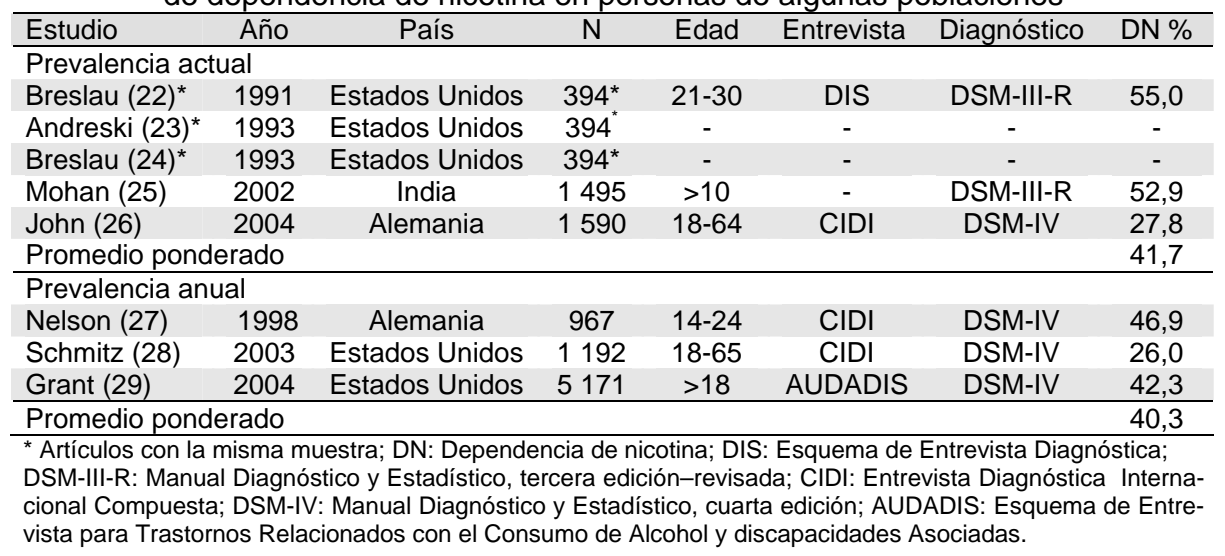


Prevalencia de dependencia de nicotina

En resumen la prevalencia actual promedio fue $41,7 \%$ y ponderada $44,7 \%$ (IC $95 \%$ 40,0-46,4). Por su parte, la prevalencia anual promedio fue 40,3\% y ponderada 37,7 \% (IC 95 \% 36,6-38,8). La prevalencia encontrada en cada estudio se muestra en la Tabla 1.

Características sociodemográficas y otros factores asociados con dependencia de nicotina

Nelson (30) informó que la prevalencia de nicotina era independiente de la edad. Schmitz (31) encontró que se presentaba con mayor frecuencia en las personas más jóvenes. Breslau (25), Andreski (26) y John (29) evidenciaron que la DN prevalecía en aquellas personas que iniciaron el consumo de cigarrillo o se hicieron fumadores regulares a una menor edad.

Breslau (25) Nelson (28), Schmitz (31) y Grant (32) hallaron que la DN era independiente del género. Schmitz (31) informó que la DN era mayor en solteros y en estratos económicos más bajos. Breslau $(25,27)$ y Andreski (26) señalaron que la DN se observaba con mayor frecuencia en las personas con menos años de escolaridad y en caucásicos que en afroamericanos.

Nelson (29), Schmitz (30) y Grant (31) encontraron una asociación significativa entre el número de cigarrillos fumados y DN, la DN era directamente proporcional al número de cigarrillo fumados por día, a mayor consumo diario de cigarrillo era mayor la frecuencia de DN.

\section{DISCUSIÓN}

En esta revisión se destaca que la DN se presenta en alrededor del $40 \%$ de los fumadores, $44,7 \%$ de los fumadores actuales y 37,7 \% de las personas que fumaron el último año. La DN fue independiente del sexo y mostró una relación importante con el número de cigarrillos fumados al día.

Los pocos estudios identificados muestran que se ha prestado menos atención a la DN que al consumo de cigarrillo (25-32). Los fumadores que reúnen criterios para DN, generalmente inician el consumo de cigarrillo más tempranamente $(25,29)$ e, igualmente, presentan el mayor riesgo para morbilidad física y mental. La mayoría de las enfermedades físicas relacionadas con el consumo de tabaco guardan una relación directa con el tiempo de exposición (17). De la misma forma, se relaciona presencia de trastornos mentales como esquizofrenia, trastorno depresivo mayor, trastorno de pánico y el 
consumo de sustancias legales e ilegales con el consumo de cigarrillo (3338). Sin embargo, los datos disponibles muestran que la asociación es más significativa y consistente con DN que con el simple consumo de cigarrillo $(39,40)$.

Se observó que la DN es tan frecuente en hombres como en mujeres. Se ha planteado que la DN es independiente del sexo y está determinada por factores genéticos (41-44).

La DN guarda una relación directa con el número de cigarrillos fumados, las personas con una dependencia más severa fumará más cigarrillos durante el día (29-31). Se presenta dependencia a una sustancia cuando se reúnen criterios para abstinencia (se presentan al suspender o reducir abruptamente la cantidad de sustancia administrada) o tolerancia a la sustancia (necesidad de dosis mayores y crecientes para obtener el efecto deseado) $(12,13)$.

Los fumadores que reúnen criterios para DN representan el mayor problema para la salud pública. Adicionalmente, las personas con DN presentan otros comportamientos de riesgo para la salud, como menor de consumo de alimentos saludables y de actividad física y mayor uso de alcohol $(45,46)$. En consecuencia, deben diseñarse programas y estrategias más agresivas y específicas para motivar el abandono de consumo de cigarrillo en las personas con DN $(23,47,48)$. Debe considerarse el consumo de cigarrillo, y en especial la DN, como un trastorno crónico recurrente con recaídas, remisiones y exacerbaciones $(23,49,50)$.

Esta revisión tiene varias limitaciones. Sólo se buscaron y encontraron pocos artículos en tres idiomas. No obstante, el mayor número de producción científica se divulga principalmente en inglés. Se encontró que la prevalencia actual era superior a la prevalencia anual. Esto se puede explicar por las características de las poblaciones en las que se realizaron las investigaciones. Ninguno de los estudios revisados informaba ambas prevalencias, actual y anual. De la misma forma, es difícil comparar los resultados debido a las diferencias en las características sociodemográficas de las poblaciones (por ejemplo, la edad) y a las entrevistas utilizadas para el diagnóstico de DN, según los criterios de la APA. Ningún estudio informó la prevalencia DN siguiendo los criterios de la OMS.

Se concluye que cerca de uno de cada dos fumadores regulares reúne criterios para DN actual y aproximadamente uno de cada tres presenta DN durante el último año. Se necesita mayor investigación de este tópico y cen- 
trar esfuerzos en promover y manejar el abandono del consumo de cigarrillos en las personas que presentan DN •

Agradecimientos. Esta revisión la financió el Instituto Colombiano para el Desarrollo de la Ciencia y la Tecnología (Colciencias). Contrato RC 401-2004 con la universidad Autónoma de Bucaramanga en el marco de una investigación sobre la prevalencia de consumo de cigarrillo y dependencia de nicotina en la población general de Bucaramanga, Colombia.

\section{REFERENCIAS}

1. Mokdad AH, Marks JS, Stroup DF, Gerberding JL. Actual causes of death in the United States, 2000. JAMA 2004; 291: 1238-1245.

2. Thun MJ, Apicella LF, Henley SJ. Smoking vs others risk factors as the cause of smoking-attributable deaths. JAMA 2000; 284: 706-712.

3. Ezzatti M, Lopez AD. Estimates of global mortality attributable to smoking in 2000. Lancet 2003; 362: 847-852.

4. Annual smoking-attributable mortality, years of potential life lost, and productivity losses-United States, 1997-2001. MMWR 2005; 54: 788-789.

5. Caraballo RS, Lee C-W. Consumo de tabaco entre los mexicanos y sus descendientes en Estados Unidos de América. Salud Publica Mex 2004; 46: 241250.

6. Jha P, Ranson K, Nguyen SN, Yack D. Estimates of global and regional smoking prevalence in 1995, by age and gender. Am J Public Health 2002; 92: 10021006.

7. Jarallah JS, Al-Rubean KA, Al-Nuaim ARA, Al-Ruhaily AA, Kalantan KA. Prevalence and determinants of smoking in three region of Saudi Arabia. Tob Control 1999; 8: 53-56.

8. Pärna K, Rahu K, Rahu M. Patterns of smoking in Estonia. Addiction 2002; 97: 871-876.

9. Posada JA, Torres Y. Estudio nacional de salud mental y consumo de sustancias psicoactivas, Colombia, 1993. Santafé de Bogotá: Ministerio de Salud; 1995.

10. Posada-Villa JA, Aguilar-Gaxiola SA, Magaña CG, Gómez LC. Prevalencia de trastornos mentales y uso de servicios: resultados preliminares el Estudio Nacional de Salud Mental. Colombia, 2003. Rev Colomb Psiquiatr 2004; 33: 241-262.

11. Hughes JR. Distinguishing nicotine dependence from smoking. Arch Gen Psychiatry 2001; 58: 817-818.

12. Organización Mundial de la Salud. Clasificación Internacional de las Enfermedades (CIE). Trastornos mentales y del comportamiento. Criterios diagnósticos de investigación. 10 Edición. Madrid: Meditor; 1993.

13. American Psychiatric Association. Diagnostic and statistical manual of mental disorders. 4th ed. Washington DC: American Psychiatric Association; 2000 . 
14. Fagerström KO, Kunze M, Schoberberger R, Breslau N, Hughes JR, Richard $\mathrm{RD}$, et al. Nicotine dependence versus smoking prevalence: comparison among countries and categories of smokers. Tob Control 1996; 5: 52-56.

15. Díaz FJ, Jane M, Salto E, Pardell H, Salleras L, Pinet C, et al. A brief measure of high nicotine dependence for busy clinicians and large epidemiological surveys. Aust N Z J Psychiatry 2005; 39: 161-168.

16. Benowitz NL, Hennigfield JE. Establishing a nicotine threshold for addiction. The implications for tobacco regulation. N Engl J Med 1994; 331: 123-125.

17. Brook JS, Brook DW, Zhang C, Cohen P. Tobacco use and health in young adulthood. J Genet Psychol 2004; 165: 310-323.

18. Fagerström KO. Measuring degree of physical dependence to tobacco smoking with reference to individualization of treatment. Addict Behav 1978; 3: 235-241.

19. Etter J-F, Le Houezec J, Perneger TV. A self-questionnarire to measure dependence on cigarette: The Cigarette Dependence Scale. Neuropharmacology 2003; 28: 359-370.

20. Posada JA. Las técnicas de evaluación psicométrica en salud mental y psiquiatría. Rev Colomb Psiquiatr 1998; 27: 143-156.

21. West R, McNeil A, Raw M. Smoking cessation guidelines for health professionals: an update. Thorax 2000; 55: 987-999.

22. Campo A. Dependencia de nicotina. Aproximación a su manejo farmacológico. Rev Colomb Psiquiatr 2002; 31: 67-72.

23. Henningfield JE, Fant RV, Buchhalter AR, Sitzer ML. Pharmacotherapy for nicotine dependence. CA Cancer J Clin 2005; 55: 281-299.

24. Campo A. ¿Cómo ayudar a los pacientes a dejar de fumar? Educ Invest Enf 2005; 23 (2): 96-103.

25. Breslau N, Kilbey MM, Andreski P. Nicotine dependence, major depression and anxiety in young adults. Arch Gen Psychiatry 1991; 48: 1069-1074.

26. Andreski P, Breslau N. Smoking and nicotine dependence in young adults: differences between blacks and whites. Drug Alcohol Depend 1993; 32: 119125.

27. Breslau N, Kilbey MM, Andreski P. Vulnerability to psychopathology in nicotine-dependent smokers: an epidemiological study of young adults. Am J Psychiatry 1993; 150: 941-946.

28. Mohan D, Chopra A, Sethi H. The co-occurrence of tobacco \& alcohol I general population of Metropolis Delhi. Indian J Med Res 2002; 116: 150-154.

29. John U, Meyer C, Hapke U, Rumpff H-J. Nicotine dependence and lifetime amount of smoking in a population sample. Eur J Public Health 2004; 14: 182-185.

30. Nelson CB, Wittchen H-U. Smoking and nicotine dependence. Results from a sample of 14-to-24 year-olds in Germany. Eur Addict Res 1998; 4: 42-49.

31. Schmitz N, Kruse J, Kugler J. Disabilities, quality of life, and mental disorders associated with smoking and nicotine dependence. Am J Psychiatry 2003; 160: 1670-1676.

32. Grant BF, Hasin DS, Chou SP, Stinton FS, Dawson DA. Nicotine dependence and psychiatric disorders in the United States. Results from the National 
Epidemiologic Survey on Alcohol and related conditions. Arch Gen Psychiatry 2004; 61: 1107-1115.

33. Jorm AF. Association between smoking and mental disorders: results from an Australian National Prevalence Survey. Aust N Z J Psychiatry 1999; 23: 245-248.

34. Ismail K, Sloggett A, De Stavola B. Do common mental disorders increase cigarette smoking? Results from five waves of a population-based panel cohort study. Am J Epidemiol 2000; 152: 651-657.

35. Lasser K, Boyd JW, Woolhander S, Himmelstein DU, McCormick D, Bor DH. Smoking and mental illness. A population-based prevalence study. JAMA 2000; 284: 2606-2610.

36. Farrell M, Howes S, Bebbington P, Brugha T, Jenkins R, Lewis G, et al. Nicotine, alcohol and drug dependence and psychiatric comorbidity. Results of national household survey. Br J Psychiatry 2001; 179: 432-437.

37. Breslau N, Novak SP, Kessler RC. Daily smoking and the subsequent onset of Psychiatric disorders. Psychol Med 2004; 34: 323-333.

38. Breslau N, Kilbey MM, Andreski P. DSM-III-R nicotine dependence in young adults: prevalence, correlates and associated psychiatric disorders. Addiction 1994; 89: 743-754.

39. Rhode P, Kahler CW, Lewinsohn PM, Brown RA. Psychiatric disorders, familial factors, and cigarette smoking: II. Associations with progression to daily smoking. Nicotine Tob Res 2004; 6: 119-132.

40. Merikangas KR, Stolar M, Stevens DE, Goulet J, Preisig MA, Fenton B, et al. Familial transmission of substance use disorders. Arch Gen Psychiatry 1998; 55: 973-9.

41. Bierut LJ, Dinwiddie SH, Begleiter H, Crowe RR, Hesselbrock V, Nurnberger JI, et al. Familial transmission of substance dependence: alcohol, marijuana, cocaine, and habitual smoking. Arch Gen Psychiatry 1998; 55: 9828.

42. Madden PAF, Heath AC, Pedersen NL, Kaprio J, Koskenvuo MJ, Martin NG. The genetics of smoking persistence in men and women: a multicultural study. Behav Genet 1999; 29: 423-431.

43. Kendler KS, Thornton LM, Pedersen NL. Tobacco consumption in Swedish twins reared apart and reared together. Arch Gen Psychiatry 2000; 57: 886892.

44. Burke V, Milligan RAK, Beilin LJ, Dunba D, Spencer M, Balde E, et al. Clustering of health-related behaviors among 18-year-old Australians. Prev Med 1997; 26: 724-733.

45. Schumman A, Hapke U, Rumpf H-J, Meyer C, Jonh U. The association between degree of nicotine dependence and other health behaviours. Eur J Public Health 2001; 11: 450-452.

46. Batra V, Patkar AA, Weibel S, Leone FT. Tobacco smoking as a chronic disease: notes on prevention and treatment. Prim Care Clin Office Pract 2002; 29: 629-648.

47. McLellan AT, Lewis DC, O’Brien CP, Kleber HD. Drug dependence, a chronic medical illness. JAMA 2000; 284: 1689-1695. 
48. Raw M, McNeil A, West R. Smoking cessation: evidence based recommendations for the healthcare system. BMJ 1999; 318: 182-185.

49. Landcaster T, Stead L, Sigaly C, Sowden A. Effectiveness of interventions to help people stop smoking: findings from the Cochrane Library. BMJ 2000; 321: 355-358.

50. Wilhelm K, Arnold K, Niven H, Richmond R. Grey lungs and blue moods: smoking cessation in the context of lifetime depression history. Aust N Z J Psychiatry 2004; 38: 896-905. 